\title{
An efficient multigrid Poisson solver
}

\begin{abstract}
In this paper, we introduce an efficient technique known as a quarter sweeps multigrid method for solving two dimensional Poisson equation with the Dirichlet boundary condition. The method with the red black Gauss-Seidel smoothing scheme is shown to be the most superior than the half- and full-sweeps multigrid methods due to Othman et at. [8] and Gupta et al. [5], respectively. Some numerical experiments are included to confirm our recommendation.
\end{abstract}

Keyword: Full-, half- and quarter-sweeps multigrid methods; Performance evaluation; Poisson equation 